\title{
Pathogenic Variability of Pyricularia grisea from the High- and Mid-Elevation Zones of Bhutan
}

\author{
Thinlay, R. S. Zeigler, and M. R. Finckh
}

First author: National Plant Protection Centre Simthokha, Bhutan; second author: Entomology and Plant Pathology Division, International Rice Research Institute, P.O. Box 933, 1099 Manila, Philippines; and third author: Department of Ecological Plant Protection, Nordbahnhofstrasse 1a, Faculty of Agriculture, International and Rural Development and Environmental Protection, University of Kassel, P.O. Box 1252, D-37213 Witzenhausen, Germany.

Current address of R. S. Zeigler: Department of Plant Pathology, 4024 Throckmorton Plant Sciences Center, Kansas State University, Manhattan 66502.

Accepted for publication 15 February 2000.

\section{ABSTRACT}

Thinlay, Zeigler, R. S., and Finckh, M. R. 2000. Pathogenic variability of Pyricularia grisea from the high- and mid-elevation zones of Bhutan. Phytopathology 90:621-628.

Thirty isolates of $P$. grisea collected from rice during a blast epidemic in 1995 in the high $(1,800$ to $2,600 \mathrm{~m})$ and middle $(1,200$ to $1,800 \mathrm{~m})$ elevations of Bhutan and 80 isolates collected from one rice cultivar from two high- and two mid-elevation sites in 1996 were analyzed for virulence. Differential varieties were indica CO39, with five near-isogenic lines (NILs) for resistance genes in the genetic background of CO39, and japonica Lijiangxintuanheigu (LTH), with five NILs for LTH. Twelve selected Bhutanese landraces also were studied. In addition, 10 blast nurseries consisting of the NIL sets, important local landraces, and representatives of international differential groups were established in the 1996 and 1997 growing seasons in the mid- and high-elevation agroecological zones. The 110 isolates were differentiated into 53 pathotypes based on the 2 NIL sets. Thirteen isolates were avirulent on all of the NILs but were compatible with some landraces. Several isolates were able to attack one of the NILs of CO39 but not CO39. These results strongly suggest that both $\mathrm{CO} 39$ and LTH possess previously unidentified resistance. The landraces were not uniform in their reactions to the isolates. When a reaction index taking into account all individual plant reactions was used, isolates that had been assigned to the same pathotype could be further differentiated, indicating that the NIL sets could not completely discriminate virulences in Bhutanese $P$. grisea populations. In the trap nurseries, disease was always present in the middle elevations, but disease was very low during July 1996 in the high elevations and only present during August and September 1997. Almost all varietal groups were more frequently attacked in the middle than in the high elevations, indicating that the virulence spectrum is wider and the conduciveness of the environment is greater in the middle elevations. Landraces from the high elevations were most susceptible, followed by international differential groups 7 and 8 . The results suggest that selection has yielded landraces with more complete and complex resistance in the more disease-conducive mid-elevation environment. At the same time, the pathogen population also possesses a wider virulence spectrum in that environment.

Additional keywords: coevolution, natural selection, Pyricularia oryzae, traditional varieties, virulence analysis.
Rice cultivation in Bhutan is confined to the southern half of the country, ranging from low elevations (150 to $1,200 \mathrm{~m}$ ) to valleys in the mid- $(1,200$ to $1,800 \mathrm{~m})$ and high-elevation $(1,800$ to $2,600 \mathrm{~m})$ agroecological zones. These areas are strongly subdivided by mountain ranges over $3,000 \mathrm{~m}$ high, and there are large differences in occurrence of rice blast, caused by Pyricularia grisea (Cooke) Sacc. (teleomorph Magnaporthe grisea). Blast occurs regularly in seedlings in the middle elevation, but it is usually observed only on adult plants in the high-elevation areas. Differences in microenvironments within Bhutan, together with the isolation of rice-growing valleys, are most likely responsible for the observed differences in blast occurrence (9).

Limited blast outbreaks have been observed in isolated valleys in the high elevations since the mid-1980s. However, in 1995, for the first time in recorded history, there was a severe blast outbreak in the high- and mid-elevation areas of western Bhutan (10). Although this outbreak was most likely due to unusual weather conditions, a significant observation was that, in general, highelevation cultivars appeared to be particularly susceptible. There-

Corresponding author: M. R. Finckh; E-mail address: mfinckh@wiz.uni-kassel.de

Publication no. P-2000-0417-02R

(C) 2000 The American Phytopathological Society fore, incorporating effective blast resistance into acceptable cultivars is now a priority in rice improvement programs targeted at high-elevations zones in Bhutan.

Prior to 1995, no work was done in Bhutan to study the rice blast pathogen population structure, and no data on the virulence structure of Bhutanese blast populations are available to date. In a study of DNA fingerprints of $P$. grisea from Bhutan, Thinlay et al. (10) found that the blast population was highly diverse. Among 71 isolates analyzed, 58 MGR586 fingerprints (haplotypes) were identified and could be grouped into 13 statistically robust clusters. These clusters have been inferred to result from descent from a common recent ancestor and, therefore, reflect genetic lineages. The largest number of haplotypes belonged to lineages BhR1 and BhR4, which comprised 36 and $21 \%$ of the collection, respectively, and almost all isolates from these lineages originated from high elevations. This result suggested that blast populations in Bhutan are subdivided geographically, but the implications of this for virulence diversity are unknown. An estimate of pathotypic differentiation and diversity of pathogen populations may be inferred from field disease severity on a set of diverse cultivars or from direct analysis of isolate genotypes and virulence.

The objectives of this study were (i) to characterize a collection of Bhutanese $P$. grisea isolates with respect to their virulence to rice lines near-isogenic (NILs) for major blast resistance genes and to popular Bhutanese cultivars; (ii) to determine whether P. grisea 
populations within and among different ecological zones differ with respect to their virulence spectra; and (iii) to identify isolates that could be used for screening germ plasm for possible release in blast-prone areas. To address these issues, we used field nurseries planted across environments and controlled inoculation of isolates selected to maximize diversity on rice lines near-isogenic for major resistance genes.

\section{MATERIALS AND METHODS}

Cultivars and NILs. Twenty-five widely grown cultivars from Bhutan were selected to represent traditional and improved cultivars from different agroecological zones (Table 1). In addition, 12 cultivars representing various blast resistance groups, as defined through international nursery studies (1), and indica CO39 and japonica Lijiangxintuanheigu (LTH) with their respective NILs (which are well characterized for their resistance) were used (Table 1). Seeds of CO39 and LTH and their NILs were provided by R. J. Nelson (International Rice Research Institute [IRRI]) and subsequently multiplied in Bhutan. Seeds of international differential varieties were provided by S. W. Ahn (IRRI).

For screenhouse inoculations, we used a total of 24 entries, consisting of the 12 NILs with known resistances and 12 Bhutanese cultivars that were selected based on their relevance to Bhutanese agriculture. In the nurseries, 19 Bhutanese cultivars, 12 NILs, and 12 international differentials were used (Table 1). In 1996, however, LTH and its five NILs had to be omitted from the nurseries due to insufficient seed supply.

Isolate collection, maintenance, and inoculations. The isolates used were collected during the 1995 epidemic from at least 15 cultivars and 19 sites in high and middle elevations and were selected based on their site of origin and restriction fragment length poly- morphism (RFLP) fingerprints to be as diverse as possible (10). In 1996, isolates were collected from the very susceptible cv. Kuchum in four experimental sites (Table 2) to determine the pathogenic variability of blast pathogen populations in specific sites on the same cultivar. Two high-elevation sites, Bechumo 1 and 2, were located in the Thimphu district less than $2 \mathrm{~km}$ from the nursery site at Chukathanka (Table 3), while the mid-elevation sites were in fields adjacent to the nursery sites of the same name.

Single-spore isolates were obtained by streaking conidia from a discrete lesion on $4 \%$ water agar containing streptomycin at $40 \mathrm{mg} / \mathrm{liter}$. Germinating conidia were transferred to rice polish agar ( $20 \mathrm{~g}$ of rice polish, $5 \mathrm{~g}$ of ordinary sugar, $20 \mathrm{~g}$ of agar, and $40 \mathrm{mg}$ of streptomycin per liter) after $24 \mathrm{~h}$. Isolates were stored on filter paper as reference isolates for repeat inoculations (3).

Plants were grown in the screenhouse in wooden boxes $(45 \times$ $50 \times 20 \mathrm{~cm}$ ) filled with soil collected from nearby rice fields. The soil was renewed for each sowing. From 20 to 25 seeds of each cultivar were sown in single rows ( 15 to $20 \mathrm{~cm}$ long) spaced $\approx 2$ to $3 \mathrm{~cm}$ apart. All 24 differentials were planted together in each box and grown for 23 to 24 days (three- to four-leaf stage) before being inoculated. Each isolate was inoculated on two sets of differentials, usually on the same day.

Inoculum preparation, inoculation, and incubation was conducted following the protocol of Bonman et al. (3). Sporulation was induced by exposing colonized petri plates to permanent fluorescent light for 4 to 5 days. Spores were washed off the plates and concentrations were adjusted to $10^{5}$ spores per $\mathrm{ml}$ with a hemacytometer, and Tween $20(10 \mu \mathrm{l} / 100 \mathrm{ml}$ of spore suspension) was added as surfactant. Inoculum was sprayed to runoff on plants in the afternoon, and plants were covered with wet burlap bags for $24 \mathrm{~h}$. The temperature in the screenhouse during test periods ranged from 11 to $33^{\circ} \mathrm{C}$. Ambient temperatures only allowed for inocu-

TABLE 1. Near-isogenic lines (NILs) and rice cultivars used as differentials in the screenhouse and trap nurseries for virulence analysis

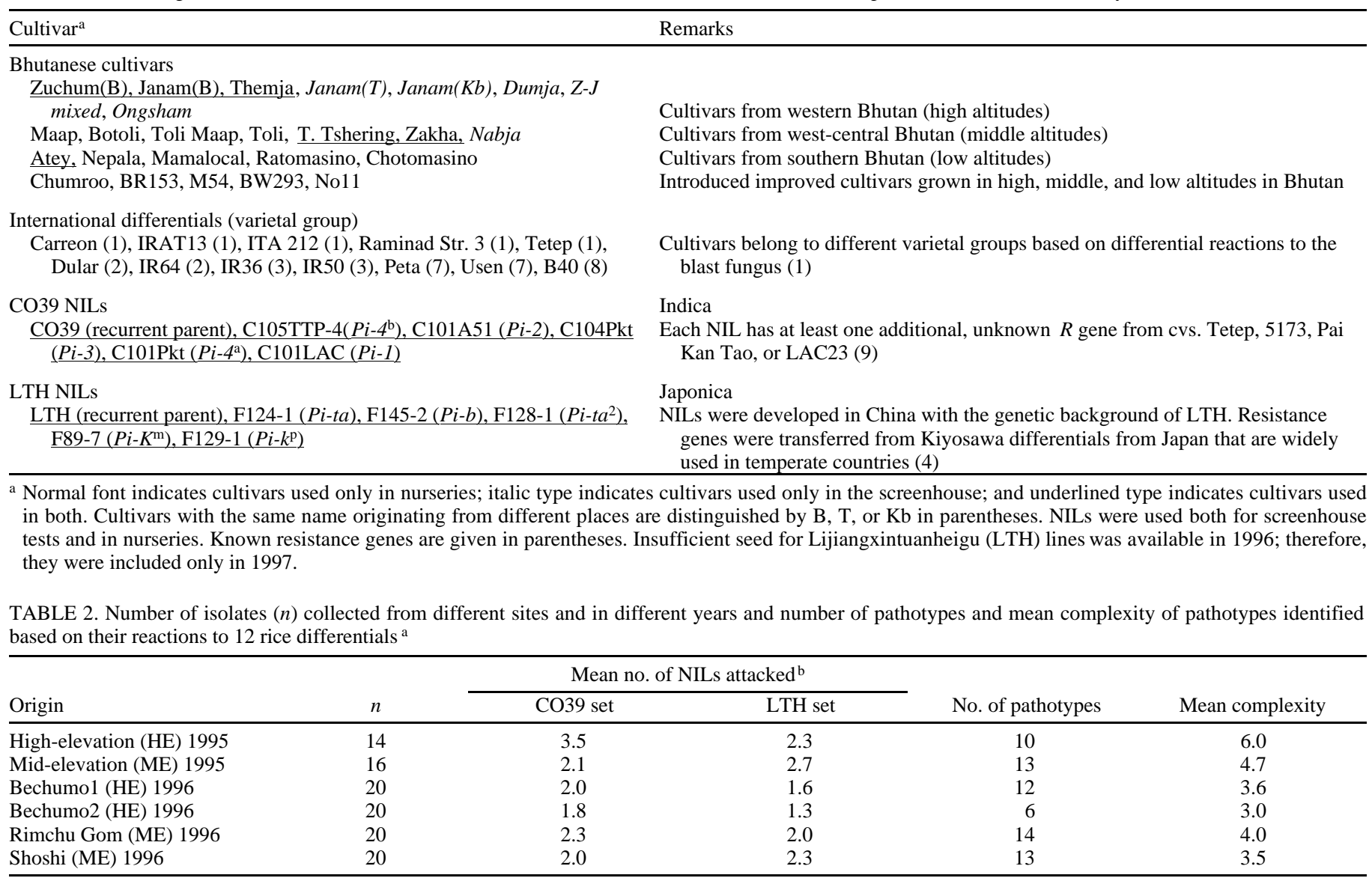

a Complexity is the mean number of differentials attacked per pathotype, calculated as the mean for all pathotypes observed per site.

${ }^{b}$ Mean of the complexity of the pathotypes identified by a given set of near-isogenic lines (NILs). LTH = Lijiangxintuanheigu line. 
lations between May and September and inoculum production capacity was limited, preventing truly replicated inoculation over time. However, 14 isolates of the 1995 collection were inoculated on the CO39 NIL set in 1996 and 1997 with identical results (9). The screenhouse was kept wet by regular sprinkling of water on the floor and plants. At 8 to 10 days after inoculation, individual plants were scored for blast reactions.

Trap nurseries. Trap nurseries were raised once in July 1996 and three times in 1997 (July, August, and September) during the rice-growing season at 10 sites (Table 3): 7 in the high elevations (Paro and Thimphu districts) and 3 in the middle elevations (Punakha district). The sites were selected based on the difference in the occurrence of blast in the past and as representing general ricegrowing environments in the high and middle elevations.

The nurseries were placed in the same fields in both years. There was no unflooded land available in the high elevations; therefore, seedlings were raised in both 1996 and 1997 in wooden boxes. A total of 15 to 20 seeds per entry were sown in 15-cm-long rows $2 \mathrm{~cm}$ apart. All 45 test cultivars were sown in one box. The seedlings were grown for $\approx 3$ weeks in the screenhouse, then exposed to field conditions for at least 2 weeks before evaluation. In the mid-elevation sites, the 1996 nurseries were raised in 1-m-long rows in plots in farmers' fields. One hundred seeds of each cultivar were planted in single rows. Susceptible cvs. CO39, Zechum, Janam, and Themja were planted between the other entries to enhance the spread of inoculum among the cultivars. In 1997, nurseries for the middle elevations were raised in boxes like those for the high elevations.

Disease assessment. Reaction of each plant in the screenhouse was evaluated 8 to 10 days after inoculation, and seedlings in the nursery were evaluated 30 days after sowing based on the IRRI standard evaluation scale (8). Plants with scores of 0 to 2 (no symptoms to nonsporulating spots) were considered resistant, those with scores of 3 (small lesions surrounded by necrosis with little sporulation) were considered intermediate, and those with scores of 4 and 5 (typical spindle- lesions, $3 \mathrm{~mm}$ or larger, sporulating heavily) were considered susceptible.

Pathotype codes. For each NIL, reactions could be clearly differentiated as resistant or susceptible. Resistant reactions were coded as 0 and susceptible reactions as 1 , resulting in a 12-digit binary code for each isolate that was converted into a 4-digit octal code as follows. First, the 12 NILs were divided into 4 groups of 3: (i) CO39, C101 A51, C105 TTP-4; (ii) C104 Pkt, C101 Pkt, C101 LAC; (iii) LTH, F89-7, F145-2; and (iv) F129-1, F128-1, F124-1 (Table 1 provides the resistance genes for each line). The first member of each group of three was assigned 1, the second 2 , and the third 4 . The assigned value was multiplied with the resistance reaction of an isolate, and the three values per group were summed. For example, if CO39 and C105 TTP-4 could be attacked, the resulting code value would be $1+4=5$. The maximum value per group was 7 . The octal code, thus, allowed us to unambiguously denote virulences to 12 differentials using 4 digits.

Reaction indices. Because mixed reactions were observed with most Bhutanese cultivars, the usual designation of an isolate being virulent or avirulent was useless. Therefore, a reaction index for each isolate-cultivar interaction was calculated. After classifying each single plant's reaction into resistant, susceptible, or intermediate ( $R, S$, and $I$, respectively), the percent $R$, I, and S reactions were multiplied with corresponding assigned values of 1,2 , and 3 , respectively, and their sum product, divided by 100 , was used as a reaction index. The index, thus, could vary between 1 (all plants R) and 3 (all plants S). If, for example, $30 \%$ of the plants tested were $\mathrm{R}$ and $70 \%$ were $\mathrm{S}$, the index is $2.4((70 \times 3+30 \times 1) / 100)$. Highly uniform $\mathrm{S}$ reactions should have indices $>2.4$ (a few plants can reasonably be expected to escape inoculation), whereas uniform $\mathrm{R}$ reactions should be very close to 1.0 , because susceptible plants in a resistant population cannot be interpreted as inoculation escapes. However, some I reactions may increase the index of resistant hosts above 1.0.

\section{RESULTS}

Virulence characterization. The reactions of the NILs in the screenhouse inoculations were generally uniform, with the majority of plants being either susceptible or resistant. The escape rate was 11 to $12 \%$ for susceptible plants, which means either that inoculum did not land on some plants or that it landed but failed to cause disease. In a few cases, some plants showed intermediate reaction types when the general reaction was resistant or susceptible, which may be due to effects of environmental variation.

In contrast to the NILs, the reactions of the Bhutanese cultivars were variable. Commonly, 10 to $50 \%$ of the plants tested were susceptible, while the rest were resistant, indicating that the cultivars were mixtures of genotypes and rendering them unsuitable for pathotyping.

Except for Bechumo 2, in which only 6 pathotypes could be identified among the 20 isolates, pathotype variability was very high, with at least 6 pathotypes per 10 isolates collected (Table 2). Based on their reactions to $\mathrm{CO} 39, \mathrm{LTH}$, and their respective NILs, the 110 isolates could be differentiated into a total of 53 pathotypes (Table 4). Although CO39 NILs differentiated 23 pathotypes among all 110 isolates the LTH NILs differentiated only 12. Each NIL, except F145-2, was attacked by at least one isolate.

TABLE 3. Sites for blast nurseries in three districts of western Bhutan and occurrence of blast in 1995 and in nurseries in 1996 and 1997

\begin{tabular}{|c|c|c|c|c|c|}
\hline \multirow[b]{2}{*}{ Site number and location } & \multirow[b]{2}{*}{ Environment $^{\mathrm{b}}$} & \multicolumn{4}{|c|}{ Blast occurrence $^{\mathrm{a}}$} \\
\hline & & 1995 & July 1996 & July 1997 & August-September 1997 \\
\hline \multicolumn{6}{|c|}{ High-elevation $(1,800-2,600 \mathrm{~m})$} \\
\hline \multicolumn{6}{|l|}{ Paro District } \\
\hline 1. Chankathang & Warm, humid & Severe & + & - & + \\
\hline 2. Tsento Nemja & Warm, humid & Severe & + & - & + \\
\hline 3. Tsendo Chukha & Warm, humid & Severe & + & - & + \\
\hline 4. Sharikodephu & Warm, humid & Moderate & + & - & + \\
\hline \multicolumn{6}{|l|}{ Thimphu District } \\
\hline 5. Charkilo & Cool, dry & Not observed & - & - & - \\
\hline 6. Jashigokha & Cool, humid & Severe & - & - & + \\
\hline 7. Chukathangka & Cool, humid & Severe & + & - & + \\
\hline \multicolumn{6}{|c|}{ Mid-elevation $(1,200-1,800 \mathrm{~m})$} \\
\hline \multicolumn{6}{|l|}{ Punakha District } \\
\hline 8. Rimchugom & Hot, humid & Severe & + & + & + \\
\hline 9. Rimchuwom & Hot, humid & Severe & + & + & + \\
\hline 10. Shoshi & Hot, dry & Variable & + & + & + \\
\hline
\end{tabular}

a + and - indicate blast detected or not detected, respectively.

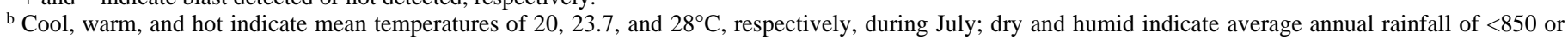
$1,200 \mathrm{~mm}$, respectively. 
The true number of pathotypes in a population can be estimated based on the number of rare pathotypes in a sample: $S=S_{\text {obs }}+$ $\left(a^{2} / 2 b\right)$, where $S_{\text {obs }}$ is the actual number of pathotypes observed in the population, $a$ is the number of pathotypes represented by only one individual (isolate), and $b$ is the number of pathotypes represented by exactly two individuals (6). This procedure relates distributions within samples and allowed us to estimate how many rare types were missed given the observed distributions and sample size. It does not require any assumption about gene frequencies or

TABLE 4. Pathotypes identified, using a set of 12 differential rice near-isogenic lines, from among 110 isolates collected in Bhutan in 1995 and $1996^{\text {a }}$

\begin{tabular}{|c|c|c|c|c|c|}
\hline \multirow{2}{*}{$\begin{array}{l}\text { Pathotype } \\
\text { octal code }^{\text {b }}\end{array}$} & \multirow{2}{*}{$\begin{array}{l}\text { No. of } \\
\text { times found }\end{array}$} & \multicolumn{2}{|c|}{ Altitude $^{c}$} & \multirow[b]{2}{*}{ Year } & \multirow{2}{*}{$\begin{array}{c}\text { No. of lines } \\
\text { attacked }\end{array}$} \\
\hline & & Middle & High & & \\
\hline 1012 & 1 & PW & $\ldots$ & 1995 & 3 \\
\hline 1032 & 2 & PW & $\ldots$ & 1995 & 4 \\
\hline 1113 & 1 & PW & $\ldots$ & 1995 & 5 \\
\hline 1510 & 1 & $\ldots$ & $\mathrm{T}$ & 1995 & 4 \\
\hline 1516 & 1 & $\mathrm{CB}$ & & 1995 & 6 \\
\hline 1710 & 1 & & $\mathrm{~T}$ & 1995 & 5 \\
\hline 1712 & 3 & $\mathrm{CB}, \mathrm{PW}$ & $\mathrm{P}$ & 1995 & 6 \\
\hline 1736 & 1 & PW & . & 1995 & 8 \\
\hline 5012 & 1 & $\ldots$ & $\mathrm{T}$ & 1995 & 4 \\
\hline 5213 & 1 & . & $\mathrm{P}$ & 1995 & 6 \\
\hline 5310 & 1 & PW & $\ldots$ & 1995 & 5 \\
\hline 5333 & 1 & $\mathrm{CB}$ & $\ldots$ & 1995 & 8 \\
\hline 5632 & 1 & $\ldots$ & $\mathrm{P}$ & 1995 & 7 \\
\hline 5710 & 3 & $\ldots$ & $\mathrm{T}$ & 1995 & 6 \\
\hline 5712 & 3 & $\ldots$ & $\mathrm{P}, \mathrm{T}$ & 1995 & 7 \\
\hline 5733 & 1 & $\ldots$ & $\mathrm{T}$ & 1995 & 9 \\
\hline 7312 & 1 & PW & & 1995 & 7 \\
\hline 7510 & 1 & $\ldots$ & $\mathrm{T}$ & 1995 & 6 \\
\hline 0012 & 2 & PW & B1 & 1995,1996 & 2 \\
\hline 0110 & 2 & $\mathrm{~S}, \mathrm{PW}^{\mathrm{d}}$ & & 1995,1996 & 2 \\
\hline 1110 & 2 & CB & B1 & 1995,1996 & 3 \\
\hline 1010 & 21 & PW, RG, S & B1, B2 & 1995,1996 & 2 \\
\hline 0000 & 13 & $\mathrm{~S}$ & $\mathrm{~B} 2$ & 1996 & 0 \\
\hline 3010 & 7 & RG, $\mathrm{S}$ & B1 & 1996 & 3 \\
\hline 5010 & 5 & RG, $S$ & B1 & 1996 & 3 \\
\hline 5211 & 4 & RG, $\mathrm{S}$ & & 1996 & 5 \\
\hline 0010 & 2 & & B1, B2 & 1996 & 1 \\
\hline 1210 & 2 & RG & $\ldots$ & 1996 & 3 \\
\hline 0030 & 1 & $\mathrm{~S}$ & $\ldots$ & 1996 & 2 \\
\hline 0111 & 1 & $\mathrm{RG}$ & $\ldots$ & 1996 & 3 \\
\hline 0311 & 1 & RG & $\ldots$ & 1996 & 4 \\
\hline 1016 & 1 & $\mathrm{~S}$ & $\ldots$ & 1996 & 4 \\
\hline 1231 & 1 & $\mathrm{~S}$ & $\ldots$ & 1996 & 5 \\
\hline 1310 & 1 & RG & $\ldots$ & 1996 & 4 \\
\hline 1410 & 1 & RG & $\ldots$ & 1996 & 3 \\
\hline 1434 & 1 & $\mathrm{~S}$ & $\ldots$ & 1996 & 5 \\
\hline 3012 & 1 & RG & $\ldots$ & 1996 & 4 \\
\hline 3031 & 1 & RG & $\ldots$ & 1996 & 5 \\
\hline 3210 & 1 & S & $\ldots$ & 1996 & 4 \\
\hline 3230 & 1 & $\mathrm{~S}$ & $\ldots$ & 1996 & 5 \\
\hline 3310 & 1 & $\mathrm{~S}$ & $\ldots$ & 1996 & 5 \\
\hline 3410 & 1 & $\ldots$ & B2 & 1996 & 4 \\
\hline 3430 & 1 & RG & & 1996 & 5 \\
\hline 5016 & 1 & $\ldots$ & B1 & 1996 & 5 \\
\hline 5110 & 1 & $\ldots$ & B1 & 1996 & 4 \\
\hline 5210 & 1 & $\ldots$ & B1 & 1996 & 4 \\
\hline 7011 & 1 & $\ldots$ & B2 & 1996 & 5 \\
\hline 7014 & 1 & $\ldots$ & B1 & 1996 & 5 \\
\hline 7031 & 1 & $\ldots$ & B2 & 1996 & 6 \\
\hline 7211 & 1 & RG & $\ldots$ & 1996 & 6 \\
\hline 7310 & 1 & $\mathrm{RG}$ & $\ldots$ & 1996 & 6 \\
\hline 7410 & 1 & $\ldots$ & B1 & 1996 & 5 \\
\hline 7414 & 1 & $\ldots$ & B1 & 1996 & 6 \\
\hline
\end{tabular}

a Total number of pathotypes is 53 , total number of times found is 110 , and mean number of lines attacked is 3.5 .

${ }^{\mathrm{b}}$ Computation of octal codes is provided in text.

${ }^{c}$ Middle altitude: $\mathrm{PW}=$ Punakha Wangdi District; $\mathrm{CB}=$ Central Bhutan; $\mathrm{S}=$ Shoshi; RG = Rimchu Gom (in PW District). High altitude: $\mathrm{P}=\mathrm{Paro} ; \mathrm{T}=$ Thimphu District; B1 and 2 = Bechumo 1 and 2, respectively, both in Thimphu District.

d The isolate from Punakha Wangdi was isolated in 1995 from a weedy host. associations. Based on the number of singleton and doubleton isolates, the estimated total number of pathotypes in the region is 159 (6).

Thirteen isolates were unable to attack any of the differentials. However, all but 1 of the 13 isolates were able to attack at least some Janam and Zuchum plants that were inoculated at the same time, as indicated by reaction indices substantially greater than 1.0 (Fig. 1A). Thus, the avirulence was not due to inadequate inoculation conditions or loss of virulence of the isolates and the isolates were assigned, accordingly, pathotype p0000 (Table 4).

The rest of the isolates were able to attack LTH; 22 isolates were unable to attack $\mathrm{CO} 39$ but were able to attack either LTH alone (p0010), LTH and one of its NILs (p0030 and p0012), or LTH and one or two CO39 NILs (p0110 attacked C104Pkt and LTH only; p0111 attacked C104Pkt, LTH, and F129-1; and p0311 also attacked C101Pkt).

Generally, isolates that were identified as the same pathotype based on their reactions on NILs were further differentiated by the Bhutanese cultivars. For example, the two p0110 isolates differed substantially in their virulence on three high-elevation landrace entries (Fig. 1B). Another example was p1010, the most frequently found pathotype, which was able to attack only CO39 and LTH. The isolates were differentiated into many different types when their reactions on Bhutanese cultivars was included (Fig. 1C).

Overall virulence frequencies were variable and ranged between 0 and $30 \%$ for NILs with known resistances. Substantial differences in virulence frequencies among high- and mid-elevation isolates were obvious only for genes $P i-K^{\mathrm{m}}$ and $P i-k^{\mathrm{p}}$. Virulences on genes $P i$-ta and Pi- $b$ were the rarest (Table 5). However, differences in virulence frequencies could not be properly analyzed statistically because of inadequate sample size.

Virulence spectra in different years and ecological zones. In the screenhouse, 77 of 110 isolates could infect only 1 to 2 differentials plus the recurrent parents (i.e., fewer than four lines were attacked; Table 4). The 14 isolates collected from the high elevations in 1995 were able to infect considerably more of the 6 CO39 lines than any other set of isolates (Table 2), with 8 isolates capable of infecting 5 of the 6 lines. The differences in complexity between high- and mid-elevation isolates in 1995 were only marginally significant at $P=0.07$, however. No such isolates were recovered from the middle elevations in 1995 or from any location in 1996. None of the isolates could infect more than four of the six LTH lines. Overall, virulence complexity was higher for the isolate collection in 1995 than in 1996 (Table 2), when 11 of 22 pathotypes found infected 6 or more of the differentials, while in 1996 only 4 of 35 pathotypes were able to infect 6 differentials. In contrast to the 1995 collection, the most complex isolates in 1996 came from Rimchu Gom from the mid-elevation area and the least complex isolates were from Bechumo 2 in the high-elevation areas. However, the differences among sites were not significant. Because of

TABLE 5. Percentage of isolates out of 54 high-elevation (HE) and 56 midelevation (ME) isolates able to attack recurrent rice parent lines CO39 and Lijiangxintuanheigu (LTH) and 5 near-isogenic lines (NILs) of each that possess known resistance genes under controlled conditions in the screenhouse

\begin{tabular}{llccc}
\hline & & \multicolumn{3}{c}{$\%$ of isolates attacking } \\
\cline { 3 - 5 } NIL & Resistance gene & HE & ME & All \\
\hline CO39 & Recurrent parent & 78 & 82 & 80 \\
C105TTP-4 & $P i-4^{\text {b }}$ & 37 & 23 & 30 \\
C101A51 & $P i-2$ & 19 & 23 & 21 \\
C104PKT & $P i-3$ & 24 & 29 & 26 \\
C101PKT & $P i-4^{\text {a }}$ & 22 & 36 & 29 \\
C101LAC & $P i-1$ & 28 & 13 & 20 \\
LTH & & 83 & 93 & 88 \\
F124-1 & Recurrent parent & 6 & 7 & 6 \\
F145-2 & $P i-b$ & 0 & 0 & 0 \\
F128-1 & $P i-t a^{2}$ & 19 & 23 & 21 \\
F89-7 & $P i-K^{\mathrm{m}}$ & 6 & 18 & 12 \\
F129-1 & $P i-k^{\mathrm{p}}$ & 7 & 20 & 14 \\
\hline
\end{tabular}


the different sampling schemes employed in the 2 years, complexities between years were not compared statistically.

Most pathotypes that were found more than twice in 1996 could usually not infect more than three of the differentials. For example, the most ubiquitous pathotype, p1010 (21 isolates), found in all places infected only CO39 and LTH. p3010, p5010, and p1210 were similar to p1010 but, in addition, could attack one of the CO39 NILs (Table 4). In contrast, the pathotypes collected two or three times in 1995 (p1032, p1712, p5710, and p5712) infected four to seven of the differentials. A notable exception was $p 5211$, able to infect five differentials, which was found four times in the middle elevations in 1996.

Virulence spectra in nurseries. Blast occurrence in the nurseries differed greatly between July 1996 and July 1997. In most high-elevation sites at Paro and Thimphu, blast was present in July 1996 but not in July 1997; in Thimphu, the presence of blast

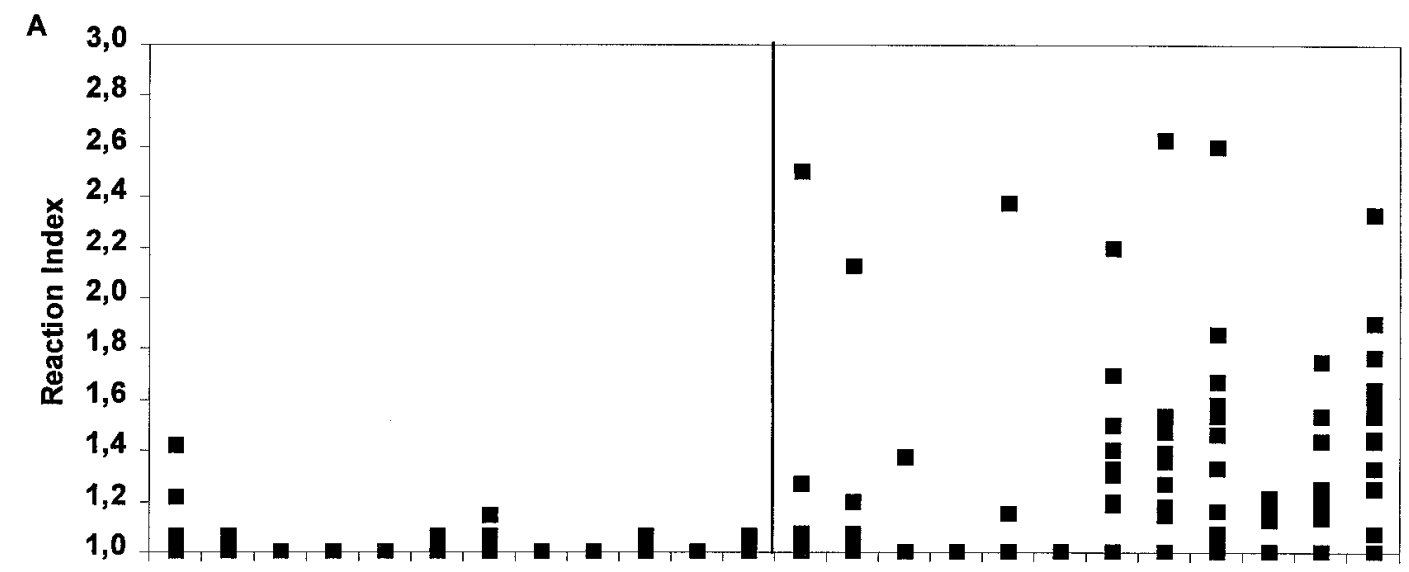

B

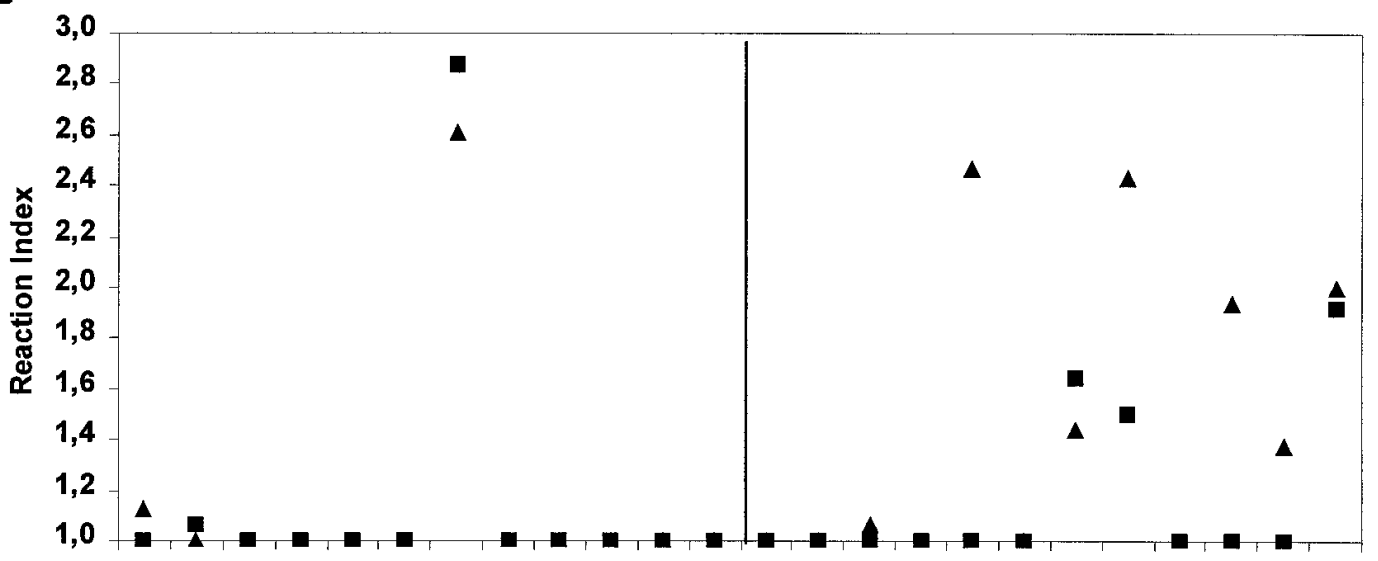

C

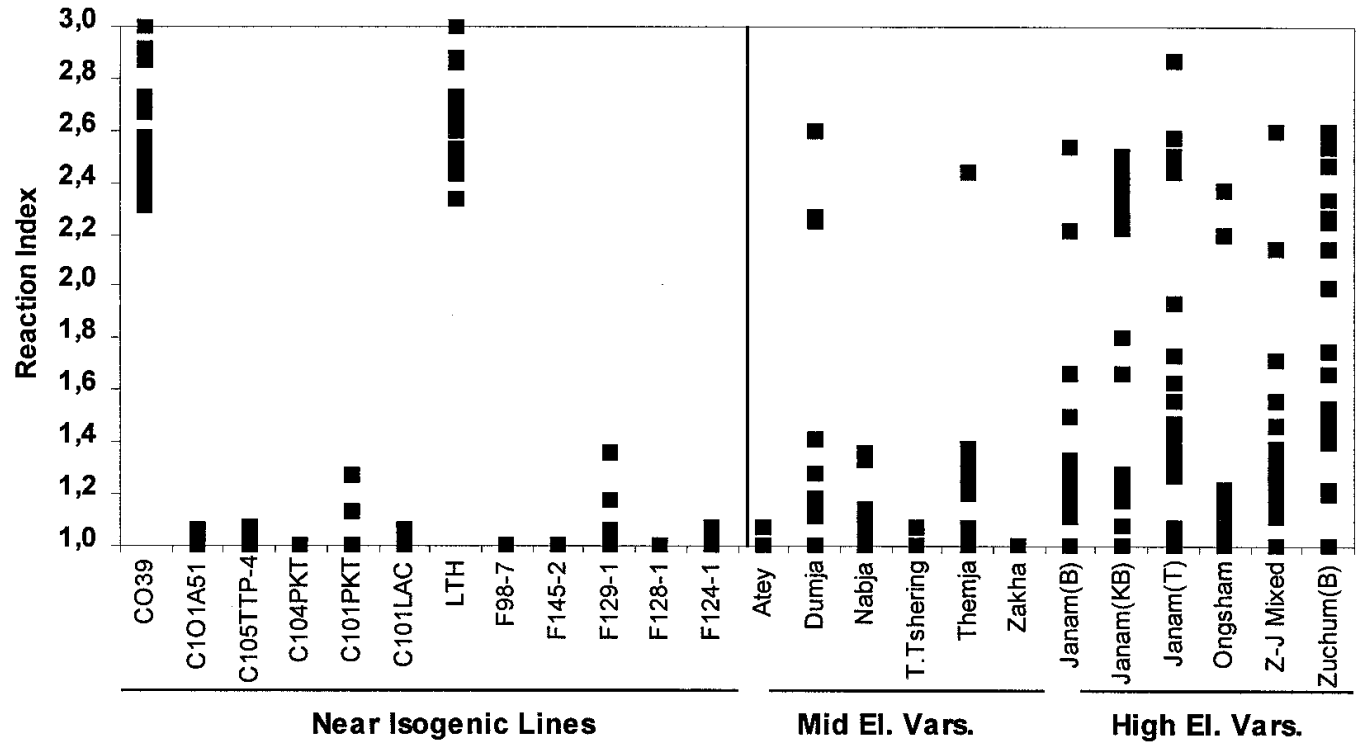

Fig. 1. Reaction indices of two sets of rice near-isogenic lines (NILs) and 12 Bhutanese cultivars to isolates assigned the same pathotype code when considering only the reactions of the NILs. Each isolate was inoculated on 30 to 50 plants per cultivar. A reaction index of $>2.4$ is considered susceptible, while indices between 1.1 and 2.2 indicate a mixed reaction to a given isolate. A, Thirteen isolates of pathotype $p 0000 ; \mathbf{B}$, two isolates of pathotype $p 0110$ (different symbols are used for each isolate); and $\mathbf{C}$, twenty-one isolates of pathotype p1010 virulent to CO39 and Lijiangxintuanheigu (LTH) but to none of the NILs. 
in both years was variable. In contrast, in Punakha, blast was present in both years at all sites and times sampled (Table 3). In the high elevations, blast occurred in all nurseries except Charkilo in August or September 1997, indicating that the blast season in the high elevations might have started later in that year. The mean number of cultivars attacked varied among sites, with the highest number of cultivars attacked in the high elevations (5.3 in Chukathangka, site 7) being as high as the lowest number in the middle elevations (5 in Shoshi, site 10) (Fig. 2). The highest number of cultivars (13.8) was attacked in Rimchu Gom (site 8), suggesting that the virulence spectrum in this site was greatest among all nursery sites. No blast was observed in Charkilo (Table 3), and only Bhutanese cultivars were attacked in Jashigokha (site 6).

Because of the very low blast incidence at high-elevation sites in July 1996, and because no late-season nurseries were set up in that year, only data from 1997 are presented in detail. There were considerable differences in the susceptibility of cultivars from each origin and varietal group depending on where they were planted (Fig. 3). For the three high-elevation cultivars, 12 of 21 (58\%) cultivar-high-elevation site interactions (Fig. 3, open bars) were susceptible, while almost $90 \%$ (16 of 18) of the interactions were susceptible in the middle elevations (Fig. 3, solid bars). In contrast, the 11 cultivars from the middle and low elevations were almost completely disease-free at all high-elevation sites, except in Thimphu, where Maap and Mama, local cultivars, were infected at one site each. Of the 11 cultivars, 9 were susceptible in at least 1 of the mid-elevation sites, mostly late in the season, while Botoli and Nepala were never attacked. Among the introduced, improved cvs. IR64 and BW293 were attacked in one high-elevation site in Thimphu, Chumroo and BR153 were resistant in all of the sites, and the other improved cultivars were susceptible in at least one of the mid-elevation sites. Infection was observed only in the late season, except for cv. No. 11, which also was infected in July (early season).

All of the Bhutanese cultivars used in the nurseries were tested against 15 to 30 isolates in a separate screenhouse test in 1996 and 1997 (9). Cvs. Zakha, Toli, T. Tshering, M54, and IR64 were resistant in the screenhouse test, but they were susceptible in some nursery sites. In contrast, cultivars such as Botoli, Nepala, BR153, and Chumroo were resistant in both the screenhouse test and in the nurseries.

Except for the recurrent parent, LTH, which was attacked in two high-elevation sites, the LTH NILs appeared very resistant in all high-elevation sites. In contrast, F145-2, F98-7, and F129-1 were attacked in one or two of the mid-elevation sites. F145-2, however, was not attacked by any of the 110 isolates tested in the screenhouse but was susceptible in the two nursery sites Rimchu Gom and Rimchu Wom. Also, in the screenhouse many isolates from the high and middle elevations readily infected the other four LTH NILs (all pathotypes with the third digit $>1$ or the fourth digit $>0$ in Table 4).

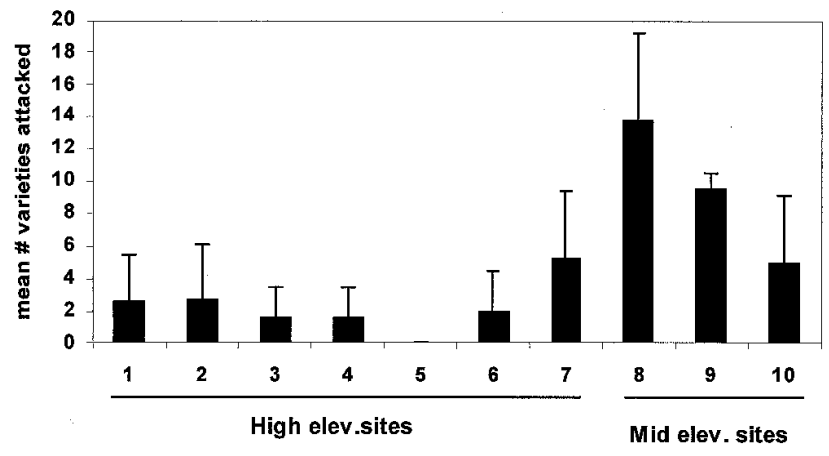

Fig. 2. Mean number of cultivars of 43 attacked in 10 trap nurseries (Table 2) set once in 1996 and three times in 1997.
All CO39 lines were infected in at least one of the mid-elevation sites, but C105TTP-4 and C101Pkt were not infected in the high elevations. However, in screenhouse tests many isolates from the high elevations could attack C105TTP-4 (all isolates with a first digit of 5 or 7 in Table 4) and C101PKT (all isolates with a second digit of 2, 3, 6, or 7 in Table 4).

The international differential varieties from groups $1,2,3$, and 7 generally were resistant in the high-elevation sites, except for Usen (group 7), which was infected in one high-elevation site. From varietal group 1, Tetep, IRAT13, and Raminad Str. 3 were attacked in one mid-elevation nursery site (Rimchu Gom) but not Carreon and ITA212. Dular and IR64 (group 2) and IR50 and IR36 (group 3) were resistant in all sites, except for IR36, which was infected in one high-elevation site. Usen and Peta (group 7) were susceptible in one or all mid-elevation sites, respectively. B40 and CO39, both belonging to group 8, were the most susceptible of the differential varietal groups, being infected each in four high-elevation sites and in one or two mid-elevation sites, respectively. As with the Bhutanese cultivars, a higher number of NILs were infected in the mid- than in the high-elevation sites. Surprisingly, IR64 collected from a Bhutanese farmer who had grown it was susceptible in Jashigokha, whereas IR64 obtained from IRRI was not. This could be due to escape. However, screenhouse inoculations of the two different seed batches indicated that the Bhutanese IR64 was not identical in resistance to the IRRI-provided seed (9).

\section{DISCUSSION}

Although isolates with the same virulences to the 12 NILs were assigned the same pathotype code, clearly the isolates were different from each other when their reactions on Bhutanese cultivars were considered (Fig. 1). Some of the isolates of the same pathotype belong to different phylogenetic lineages or have different haplotypes within a lineage. Pathotypic differences became even more evident when some isolates were inoculated onto more than 70 Bhutanese cultivars (9). Thus, the fact that the same pathotypes were sometimes collected from different regions and years cannot be taken as evidence for population overlap. For more detailed work, it would be necessary to develop a well-defined Bhutanese differential set based on Bhutanese materials that are selected to be homogeneous for their reactions to blast. These would presumably better probe the blast population in Bhutan, because isolates are adapted to Bhutanese cultivars. The NILs are still an effective tool because the $R$ genes are well known.

Within three 1996 collection sites, 11 to 14 pathotypes per site could be identified among 20 isolates per site (Table 2). In contrast, in Bechumo 2 in the high elevations, only six pathotypes were distinguished, with the most prevalent pathotype (p0000) being avirulent to all differentials. Unfortunately, cv. Kuchum, from which the isolates in 1996 were collected, was accidentally omitted from the screenhouse tests, making it impossible to determine if loss of virulence had occurred.

The low diversity at Bechumo 2 could be due to several factors. Host diversity can influence pathogen diversity but, in this particular case, the site is only 100 to $200 \mathrm{~m}$ from Bechumo 1 , where almost the same cultivars are cultivated as at Bechumo 2. Kuchum seed had been provided by us and originated from one seed batch. All isolations were made from Kuchum in all of the sites; therefore, the difference in virulence diversity could not have been due to differential host resistance. The remaining plausible explanation could be differences in microenvironmental conditions. Bechumo 2 is a well-exposed and aerated site and receives maximum sunlight. The neck blast incidence in this site in 1996 was $\approx 30 \%$, with a severity of $\approx 5$ to $10 \%$. In contrast, Bechumo 1 is surrounded by trees and boulders that act as natural barriers for wind and sunlight. This site remains relatively wet until late morning, and blast occurs every year. In 1996, neck blast incidence was 
between 40 and $45 \%$, with a severity of $\approx 45 \%$. Thus, microenvironmental differences between the sites could have affected the survival and population size of the pathogen.

There was no definite pattern to the occurrence of pathotypes in different collection sites. For instance, $p 1010$, which was recovered 21 times, was found in almost all collection sites; p0000, which occurred 13 times, was found in Bechumo 2 and Shoshi.

Relatively more pathotypes were detected among the 30 isolates from the 1995 collection (21 pathotypes and 30 isolates) than among the isolates collected in 1996 (33 pathotypes and 80 isolates); only 4 pathotypes ( $1010, p 1110, p 0110$, and p0012) were shared between the two different collection years (Table 4). Most likely, more pathotypes were detected among the 1995 isolates because they were collected from 14 cultivars and from 19 sites and preselected based on DNA fingerprint diversity. In contrast, in 1996, all isolates were collected from one cultivar and from only four sites. The most complex pathotypes are from the 1995 high-elevation collection, where severe blast outbreaks had occurred in that year (10). Thus, host diversity and conducive environments could have contributed to the variability and complexity of the pathogen population in 1995 .

One of the p0110 isolates originated from a weedy host in $\mathrm{Pu}-$ nakha Wangdi in 1995. Unfortunately, no records as to the identity of the host are available. The two p0110 isolates performed with marked differences on four of the Bhutanese cultivars (Fig. 1B) and, in later tests on even more mid-elevation cultivars (9), indicating that they differ in virulence. It is not clear if the isolate obtained from the weed is indeed able to infect any genotype of this weed, or if it was only a chance infection due to the conducive environment and heavy inoculum pressure during the epidemic in 1995. Opportunist infection of very young seedlings have been reported for some isolates that cannot reinfect the plants as they develop beyond the seedling stage (4).

Trap nurseries are an effective tool to monitor changes in virulence spectrum in different locations and to study seedling resistance (1). Nurseries also give information about disease pressure at the time of evaluation. The advantage with this method is that very little equipment and labor are required. Within nurseries, an estimate of the virulence spectrum of populations can be obtained. However, neither pathotypes nor virulence frequencies can be determined. There also are problems of virulence spectrum representation because infrequent virulences within populations may not be detected if nurseries are not set continuously. Also, in the field, an individual cultivar is exposed to a range of pathogen isolates consisting of individuals that may or may not be clones of each other, and induced resistance may hinder later attack even by virulent pathotypes. Thus, any inference about virulence diversity of a pathogen population should be made from inoculations of isolates on well-defined differential lines carrying different resistance genes and under a controlled environment. Nonetheless, our nurseries clearly demonstrated that pathogen populations differed in virulence spectra among sites.

In the mid-elevation nursery sites in 1997 , the pathogen population could attack many more cultivars than in the high elevations, suggesting that the population virulence spectrum is generally broader in middle than in high elevations. In the mid-elevation sites, the conditions remain warm and humid throughout the year whereas, in the high elevations, the early part of the year is cold and dry. Only from July through September are conditions favorable for blast development in the high elevations. The year-round conduciveness to blast most likely helps to promote diversity for virulence in the middle elevations because it allows for more disease cycles, leading to a larger population with increased possibilities for mutation. It also is possible that host diversity may contribute to pathogen diversity through differential adaptation. In general, there are more rice cultivars grown in the mid-elevation areas than in the high elevations. In high elevations, fewer cultivars are grown due to limited availability of materials adapted to cold conditions (5). We also have shown that, at least in mid-elevation cultivars, a certain degree of outcrossing is taking place and that the diversity for resistance within Bhutanese landraces is not only due to mixing of different genotypes (9).

The 13 p0000 isolates that were avirulent on all NILs but clearly pathogenic (Fig. 1A) strongly suggest that both CO39 and LTH carry previously unidentified resistance genes. $\mathrm{CO} 39$, the recurrent parent of the NILs, was considered to be a highly susceptible cultivar (7), but it was not infected in one nursery site in the middle elevation, while two of its isolines (C104Pkt and C101Pkt) were infected. In the field, lack of infection is not necessarily a sign of resistance because of the possibility of disease escape. However, Yamamoto and Ogawa (12) already have reported that CO39 showed partial resistance to some Japanese isolates. CO39 was also shown to carry at least one gene similar to $P i-a$ that conditioned complete resistance to some Japanese and Philippine isolates $(7,12)$, and it was resistant to all Philippine isolates belonging to lineage 1 (4). The presence of at least one additional resistance gene in CO39 was supported by our screenhouse tests, where three isolates (pathotypes p0110 and p0111) were avirulent on CO39 but virulent on C104Pkt (gene Pi-3) and one isolate (pathotype p0311) also was virulent on C101Pkt (gene $\mathrm{Pi}-4^{\mathrm{a}}$ ) (Table 4).

Differences in the reaction of a specific cultivar in a screenhouse and in the field suggest that resistance screening should consider a combination of the two evaluation methods. For example, none of the LTH NILs were attacked in the high-elevation nursery sites, but high-elevation isolates attacked most isolines in the screenhouse. It could be that field resistance in the LTH NILs is more effective in the high-elevation sites, and the LTH material could be useful for use in resistance breeding work there. The LTH NILs are japonica types that are more likely to be adapted to Bhutanese high-elevation conditions, because LTH is known for its cold tolerance in China and Japan. LTH NIL F145-2 might be a suitable donor for resistance, particularly in the high elevations of Bhutan, because it was not attacked either in the screenhouse or in the high-elevation nurseries. It would be worthwhile to test the LTH NILs further in more environments in the high elevations of Bhutan.

Some of the most complex isolates certainly would be the best choice when trying to determine representative isolates. However, because of the limited variation in virulence on the NIL differentials and the large variation in resistance within Bhutanese landraces (9), it is difficult to recommend a small set of isolates. There are practical limits to the number of isolates that can be tested in the screenhouse due to cost, time, and space. In the Bhutanese situation, it is always difficult to collect a representative set of isolates from different agroecological zones for testing in the screenhouse due to the complexity of the environment and to inacces-

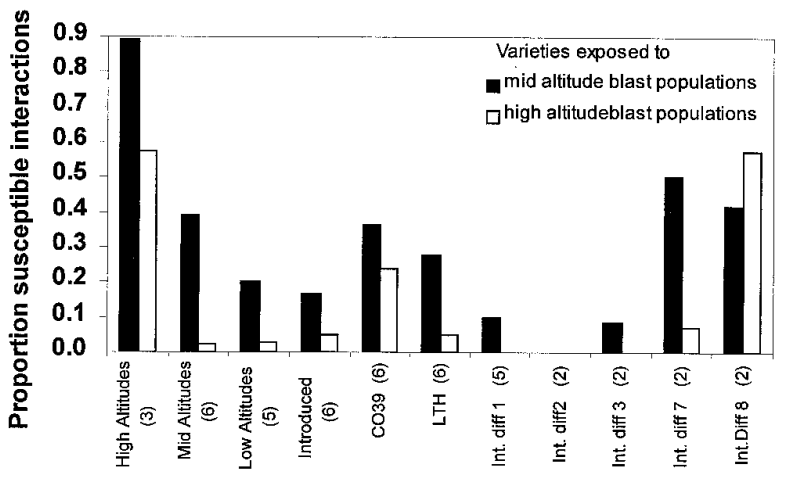

Varietal group

Fig. 3. Frequency of times plants from different varietal groups (Table 1) were infected in three trap nurseries set three times when blast occurred in 1997 in the middle elevations (solid bars) and in seven trap nurseries set one time when blast occurred in the high elevations (open bars). The number of cultivars used in each group is given in parentheses. 
sibility of many places. Hence, the trap nursery method offers an effective preliminary method to screen cultivars in different environments with minimal cost and time involved. The nursery method requires minimum equipment, yet appears to be effective in describing composite virulence spectrum and, thus, is useful for monitoring pathogen populations over time and space. However, a given trap nursery site may not represent the overall rice area, and the importance of placing several nurseries throughout the season is highlighted by the differences in the occurrence of blast between July 1996 and July 1997 that were most likely due to weather. A similar pattern between seasons has been noticed in Japan and such changes in infection patterns also were partly attributed to differences in climatic conditions, especially temperature (11).

Based on similarities in the performance of cultivars among 37 sites in 19 countries, Ahn (1) grouped the locations into four major groups. The pattern of reactions of the international differential cultivars in the different nurseries indicate that the middle and high elevations in Bhutan may be similar to location group 1 (1), where mostly japonica or basmati rice types are grown. This is very much in agreement with the classification of rice cultivars in Bhutan based on isozyme analysis. Chhetri (5) found that highelevation Bhutanese cultivars possessed increasing japonica characteristics with increasing elevation of origin, while the number of indica characteristics increased with decreasing elevation, and cultivars from low or tropical regions of Bhutan were classified as indica types. Basmati rices are indica rices that are grown in the mid-Terai regions of India and Nepal, where the environment is similar to the Bhutanese middle elevations.

Although international differential varietal group 1 is classified as broad-spectrum resistant (2), three cultivars from the group were susceptible in one site in the middle elevation. On the other hand, cultivars belonging to varietal group 7 are classified as very susceptible (1) but were susceptible only in the middle elevations. Group 8 cultivars that are classified globally as most susceptible were as susceptible as the high-elevation cultivars in the high elevations but were somewhat more resistant in the middle elevations. In Bhutan, cvs. Dular and IR64 (belonging to group 2) and IR50 and IR36 (belonging to group 3) were almost never attacked, while according to Ahn and Seshu (2) these cultivars are relatively susceptible in the major indica rice-growing international rice blast nursery sites. This may suggest that the virulence spectrum of the blast population in Bhutan is quite different from places where the above cultivars were tested. Although the lineage resistance spectrum of IR50 and IR36 has been reported to be the same (13), IR36 was attacked in one site in the middle elevations but IR50 was not. These cultivars are suspected to differ substantially in their quantitative trait loci (QTL) for blast resistance (14). In Bhutanese rice, QTL-mediated resistance has yet to be elucidated.

Our results have important implications for resistance breeding and selection in Bhutan. First, breeding for resistance must take into account the variability of pathogen populations in different sites. Cultivars developed for resistance in one site may not be effective in other environments. Second, breeding and selection for resistance should be done in areas of high blast pressure and variable pathogen population.

There is a need to develop a differential host set including Bhutanese cultivars to elucidate the diversity and relevant viru- lence spectrum of the blast population in different rice-growing areas. In the immediate future, the best choice might be to use broad virulence spectrum isolates from different places that also represent a diversity of DNA fingerprints to screen resistance in Bhutanese cultivars. However, isolates from southern and eastern parts of the country have to be included to assure a broader nationwide representation. Although the Bhutanese cultivars are not suitable to determine an isolate's virulence, they are still useful to demonstrate differences among isolates.

\section{ACKNOWLEDGMENTS}

The research described in this paper has been supported by the Swiss development Cooperation through arrangement with the Swiss Federal Institute of Technology in Switzerland and the International Rice Research Institute in the Philippines. We are grateful to the Ministry of Agriculture, Royal Government of Bhutan, for support in the field work.

\section{LITERATURE CITED}

1. Ahn, S. W. 1994. International Collaboration on Breeding for Resistance to Rice Blast. Pages 137-153 in: Rice Blast Disease. R. S. Zeigler, S. A. Leong, and P. S. Teng, eds. International Rice Research Institute, Manila, Philippines.

2. Ahn, S. W., and Seshu, D. V. 1987. Geographical variation of virulence patterns of the rice blast fungus. Page 152 in: Abstr. 11th Int. Congr. Plant Prot. International Rice Research Institute, Manila, Philippines.

3. Bonman, J. M., Vergel de Dios, T. I., and Khin, M. M. 1986. Physiologic specialization of Pyricularia oryzae in the Philippines. Plant Dis. 70:767769.

4. Chen, D., Zeigler, R. S., Leung, H., and Nelson, R. J. 1995. Population structure of Pyricularia grisea at two screening sites in the Philippines. Phytopathology 85:1011-1019.

5. Chhetri, G. B. 1992. An analysis of morpho-agronomic traits, isozyme polymorphism and cross compatibility of traditional rices (Oryza sativa L.) of Bhutan. M.Sc. thesis. University of the Philippines, Los Banos.

6. Colwell, R. K., and Coddington, J. A. 1995. Estimating terrestrial biodiversity through extrapolation. Pages 101-118 in: Biodiversity Measurement and Estimation. D. L. Hawksworth. ed. The Royal Society and Chapman and Hall, London.

7. Inukai, T., Nelson, R. J., Zeigler, R. S., Sarkarung, S., Takamure, I., and Kinoshita, T. 1994. Differentiation of pathogenic races of rice blast fungus by using near-isogenic lines with Indica genetic background. Fac. Agric. Hokkaido Univ. 66:27-35.

8. IRRI. 1996. Standard Evaluation System for Rice. International Rice Research Institute, Manila, Philippines.

9. Thinlay. 1998. Rice blast, caused by Magnaporthe grisea, in Bhutan and development of strategies for resistance breeding and management. Ph.D. thesis. ETH, No. 12777. Swess Federal Institute of Technology, Zurich.

10. Thinlay, Finckh, M. R., Bordeos, A. C., and Zeigler, R. S. Effects and possible causes of an unprecedented rice blast epidemic in the traditional farming system of Bhutan. Agric. Ecosyst. Environ. In press.

11. Yamada, M. 1979. Distribution and population change in races of rice blast fungus, Pyricularia oryzae, in Japan. Rev. Plant. Prot. Res. 12:64-79.

12. Yamamoto, Y., and Ogawa, T. 1991. Resistance of a rice indica cultivar CO39 to blast in Japan. Jpn. J. Breed. 41(Suppl. 2):416-417.

13. Zeigler, R. S., Cuoc, L. X., Scott, R. P., Bernardo, M. A., Chen, D. H., Valent, B., and Nelson, R. J. 1995. The relationship between lineage and virulence in Pyricularia grisea in the Philippines. Phytopathology 85:443451.

14. Zeigler, R. S., Guico, E., Scott, R. P., and Nelson, R. P. 1996. Relationship between partial resistance and susceptibility of rice cultivars to lineages of Pyricularia grisea. Oryza 33:273-281. 